\title{
La República de Corea: cultura, globalización y cambio social
}

Nayelli López Rocha*
Andrii Ryzhkov**

\section{RESUMEN}

La República de Corea ha logrado posicionarse mundialmente como un país de rápido desarrollo, de economía fuerte, caracterizado como un actor diligente, que participa activamente en la dinámica global. En las últimas décadas ha fungido como un país "modelo" bajo el discurso de haber logrado una rápida transformación: salió de las cenizas para convertirse en un país donante en un periodo récord. Aunque esta transformación se debe en gran medida al proceso democratizador y a la fuerza que trajo la liberalización de los mercados mundiales, en este trabajo se analiza cómo el país asiático ha enfrentado el proceso de la globalización pero desde la estructura social. Se indaga por las implicaciones que tiene en la sociedad coreana y su cultura la inserción del país al proceso de globalización, además de las manifestaciones sociales que en la actualidad revelan escenarios de choque y conflicto entre los diferentes grupos de la sociedad.

Palabras clave: República de Corea, cultura coreana, globalización, cambio social, sociedad coreana.

\section{Republic of Korea: culture, globalization and social change}

\footnotetext{
Doctora en Estudios Coreanos. Profesora-investigadora, Universidad Autónoma de Nayarit (México). [nayelli. lopezr@uan.edu.mx].

** Doctor en Lenguas de Asia y África con especialidad en lengua coreana. Universidad Autónoma de Nayarit (México). [andrii.ryzhkov@uan.edu.mx].

Recibido: 17 de febrero de 2017 / Modificado: 21 de marzo de 2017 / Aceptado: 3 de abril de 2017

Para citar este artículo:

López Rocha, N. y Ryzhkov, A. (2017). La República de Corea: cultura, globalización y cambio social. OAsıs, 26, 123-141. Dor: https://doi.org/10.18601/16577558.n26.08
} 
rapid transformation out of the ashes in order to become a donor country in a short period. Although this transformation is mainly due to the democratization process and the order imposed by the liberalization of world markets, this paper analyzes how the Asian country has faced the process of globalization, focusing mainly on the social structure. The article explores the impact that the integration of the country into the globalization process has had on the Korean society and culture, as well as the social manifestations that currently reveal scenarios of confrontation and conflict between different groups inside the country.

Key words: Republic of Korea, Korean culture, globalization, social change, Korean society.

\section{INTRODUCCIÓN}

La República de Corea ${ }^{1}$ es un país que, desde el discurso oficial y ante los ojos de la comunidad internacional ${ }^{2}$, ha logrado insertarse de manera óptima al proceso de modernización que se llevó a cabo desde las últimas décadas del siglo pasado. Empujado por la liberación de las prácticas económico-capitalistas avivadas por la interconexión mundial, Corea del Sur logró ejecutar un desarrollo económico acelerado que lo insertaría de manera casi simultánea a los procesos que desencadenó mundialmente la globalización. Esto lo forzó a satisfacer las demandas que este proceso mundial exige a los Estados y sus comunidades, para convertirse en países confiables y aptos para la interacción comercial y la negociación en la arena internacional. Así, el país asiático tuvo que enfrentar de manera vertiginosa una rápida adaptación estructural, no solo hacia el exterior, sino también hacia el interior.

El proceso para lograr el desarrollo económico de Corea, iniciado en la década de los sesenta del siglo anterior, no es más una sorpresa para la comunidad internacional en la actualidad (Nelson y Pack, 1999). Corea ha sido identificada por los imaginarios públicos en las últimas dos décadas, como un país que ha sabido aprovechar, no solo los recursos humanos y tecnológicos, sino también su política exterior, escalando en espacios de injerencia internacional, posicionándose en áreas estratégicas para la toma de decisiones en el ámbito mundial a través de su presencia en diversos organismos internacionales como la Organización de las Naciones Unidas (ONU), la Organización para el Desarrollo Económico (ocDE) o el G-20 (Nelson y Pack, 1999).

Esta habilidad para impactar en el espacio internacional, también le ha permitido transmutar para priorizar sus intereses en las últimas décadas y, junto con ello, reestructurar las es-

1 En adelante se hablará indistintamente de la República de Corea como Corea o Corea del Sur.

2 Según el Banco Mundial (2017), "La experiencia de Corea en el desarrollo sostenible, proporcionando infraestructura y mejores servicios para mejorar la vida de las personas, y su transición hacia una economía del conocimiento dinámico, brinda experiencias que pueden beneficiar a muchos otros países en desarrollo” (The World Bank, 2017). 
trategias para llegar a diversas sociedades en las que Corea ha puesto su interés, principalmente para el intercambio económico.

Desde esta perspectiva, los procesos de modernización y de globalización de Corea, que en su momento implicaron el proceso democratizador ${ }^{3}$ del país, se pueden catalogar como exitosos pero, ¿cómo ha enfrentado Corea el cambio sociocultural que se requiere para mantener el proceso de desarrollo en la nueva dinámica globalizadora? Pues uno de los grandes cuestionamientos del "Milagro coreano" es precisamente la brecha que se generó entre el desarrollo social y el desarrollo económico del país.

Para lograr algunas respuestas a este interrogante, en este trabajo se analiza la manera en la que la República de Corea ha enfrentado los cambios socioculturales que el proceso de globalización requiere. Se explora también si estas transformaciones coinciden con la imagen que se promueve a través de su política exterior donde se enfatiza, en diversos niveles, su imagen como un país moderno, exitoso, desarrollado, etc., que pueda, en palabras de Samuel Koo, llegar a ser uno de los países líderes más importantes ${ }^{4}$.
Para sustentar esta investigación se hará una revisión del concepto de globalización aproximándonos a las definiciones más generales que discuten las características y las dimensiones de este, tomando en cuenta el concepto que definió al proceso globalizatorio de Corea en su momento. Posteriormente, se indaga cómo este fenómeno mundial ha impactado en la República de Corea, principalmente sus implicaciones en la sociedad coreana, a partir de una reflexión acerca del impacto de la implementación de una política globalizatoria dos décadas atrás. Para hacer este estudio se analizan textos que han explicado desde diversas perspectivas la globalización y, específicamente, el caso de la República de Corea. La investigación también se sustenta en información recopilada en trabajo de campo y la observación participante aplicada en este país ${ }^{5}$. Esto servirá para establecer un diálogo entre el posicionamiento formal, desde el Estado, y los eventos en la realidad de la sociedad coreana que evidencian el impacto del proceso de la globalización en la Corea actual.

3 Según Chaibong Hahm (2008) es en 1987 cuando se logra la transición democrática en Corea -en el sentido de elección presidencial directa-. Sin embargo, es desde 1998 con la elección de los presidentes Kim Dae Jung (1998-2003), Roh Moo Hyun (2003-2008) y Lee Myung Bak (2008-2013) que la democracia coreana llega a su total madurez.

4 Para más información sobre el Consejo Presidencial para la Marca País de la República de Corea y su misión para la mejora de la imagen de Corea en el exterior, consultar: http://17koreabrand.pa.go.kr/gokr/en/cms/selectKbrdCmsPageTbl.do?cd $=0124 \& \mathrm{~m} 1=2 \& \mathrm{~m} 2=1$.

5 Los autores de este trabajo han vivido en la República de Corea por lapsos de 11 y 5 años. Durante estos periodos, el perfil profesional de ambos permitió recoger información de primera mano desde la cotidianidad de la sociedad y la cultura de Corea. 


\section{EL PROCESO DE GLOBALIZACIÓN}

El discurso de la globalización en el periodo contemporáneo, más allá de la definición del concepto, rescata diferentes perspectivas que intentan definir los orígenes y el desarrollo de este fenómeno mundial, que está presente en la vida de los habitantes del mundo, pero que aún no se puede caracterizar del todo.

Existen posicionamientos que evidencian la dificultad para coincidir en la definición de la globalización, como afirma Serna (2012). El discurso de la globalización tiene coincidencias en el sentido de que esta "es", perspectiva afirmada por Batelson (citado en Serna, 2012), y compartida por García Canclini (1999), quien asevera que la mayoría de los estudios confirman su existencia, pero no coinciden ni se ponen de acuerdo en lo que la globalización "es", es decir, se confirma que existe, pero no se coincide aún en una definición.

Serna (2012) también expone en su libro, que Goran Therborn identifica la existencia de cinco discursos sobre la globalización que predominan tanto en la academia como en la discusión periodística, a saber:

A. Discurso de la economía y la competitividad. B. Discurso sociocrítico. C. Discurso de la (im)potencia del Estado. D. Discurso cultural y E. Discurso de la ecología planetaria. Therborn conceptualiza a la globalización como: tendencias hacia el alcance mundial, impacto o interconexión de los fenómenos sociales o hacia una conciencia mundial entre los actores sociales. Therborn prefiere hablar de globalizaciones, reconociendo los múltiples procesos sociales implícitos en este fenómeno mundial.
Lo que señala el autor es que debe aceptarse que la idea de que el mundo entero ha estado inmerso en un proceso creciente de integración no es precisamente novedosa pues desde el siglo xIX, autores como Carlos Marx observaron cómo el capitalismo estaba alcanzando niveles mundiales (Serna, 2012).

Una de las reflexiones más interesantes que nos proporciona el trabajo de Serna (2012) es la que hace sobre Emmanuel Wallerstein, quien enfatiza el uso de la categoría del Estado-nación por excelencia para analizar las problemáticas que sucedían en el mundo. La aportación de Wallerstein es establecer una nueva categoría para el análisis de los fenómenos sociales a partir de la noción de sistemasmundo que enfatizan, en palabras de Serna, las formas de organización social de alcance transnacional (2012).

Entendemos, entonces, que la noción de la globalización ha existido desde tiempos antiguos, previos a la era moderna y, consecuentemente, posmoderna, reflexión que también rescata Ianni (1996), al explicar que "Desde que el capitalismo se desarrolló en Europa, siempre presentó connotaciones internacionales, multinacionales, transnacionales, mundiales, desarrolladas en el interior de la acumulación originaria, del mercantilismo, el colonialismo, el imperialismo, la dependencia, la interdependencia”.

Lo interesante de esta revisión es entender las categorías de análisis que se utilizan para describir los fenómenos sociales que están inevitablemente interconectados ante la dinamización que genera la globalización. Esta revisión nos permite también entender que la globalización puede ser analizada desde 
diferentes aproximaciones, y en este espacio se dará prioridad a enfatizar su impacto en la estructura sociocultural de cada grupo humano, sin dejar de entender que la repercusión de otras categorías, como la económica o política, influye de una u otra manera en la forma en la que se aproxima al estudio de la globalización.

Por ello, en este trabajo se retoma esta perspectiva social y, consecuentemente, cultural del proceso globalizatorio, a partir del análisis del proceso de globalización en la República de Corea. Se enfatizará el rol del Estado como la parte estructural de la nación coreana, y se reflexionará sobre el rol de este en la integración del país y su sociedad al proceso globalizatorio.

Se busca demostrar que al enfrentar los procesos de globalización, la República de Corea ha priorizado el desarrollo de ciertos sectores como el económico, el tecnológico y el político, sectores que van de la mano con la estructura del Estado, pero que la estructura sociocultural no ha sido constituida de una manera integral. Este desarrollo unilateral evidencia problemáticas sociales, minimizadas desde el discurso oficial del Estado pues empañan el proceso de desarrollo con el que tan a menudo se le asocia actualmente a la República de Corea en el contexto mundial.

Para entender estas tendencias unilaterales del proceso globalizatorio de Corea indagaremos: ¿cómo ha enfrentado la República de Corea los procesos sociales y culturales que la inserción a la globalización implica?, y ¿cuáles son las expresiones dentro de la sociedad que evidencian conflicto y desacuerdo social al proceso globalizador? En el siguiente apartado se aborda, entonces, la globalización coreana desde la perspectiva sociocultural.

\section{CULTURA, GLOBALIZACIÓN Y CAMBIO SOCIAL}

Para poder confirmar los supuestos planteados en la primera parte de este trabajo, en este apartado se abordará el análisis de diversos conceptos que permitirán reflexionar sobre la sociedad surcoreana ante los procesos de globalización en el periodo actual, es decir, se abordará este concepto pero delimitándolo a las discusiones que se dan en torno al fenómeno, y sus relaciones con lo social y lo cultural.

Brunner (citado en Ponce, 1998) señala que las sociedades contemporáneas se enfrentan a procesos de transformación abrazados por la globalización. Nos dice que "mientras el concepto de globalización procura dar cuenta de la novedad de un capitalismo que ha extendido sus límites hasta los confines del planeta, envolviéndolo en la lógica de los mercados y las redes de información, la idea de posmodernidad pretende expresar el estilo cultural correspondiente a esa realidad global”. Agrega Brunner que la realidad cultural posmoderna-descentrada, movible, hecha de múltiples fragmentos se constituye. en "autorreflexiva y muchas veces irónica de sí misma” (Ponce, 1998).

Por su parte, Gibernau (1996) define a la globalización como "la intensificación de las relaciones sociales en todo el mundo que unen localidades distintas de tal manera que lo que sucede en lo local está moldeado por eventos que ocurren a millas de distancia y viceversa”, y enfatiza la interconexión que existe entre los 
espacios locales y lo foráneo o no local, y el impacto que esta interconexión genera en los diversos espacios de contacto social, cultural, virtual, etc.

Nos dice Guibernau que se puede hacer referencia a la globalización desde tres perspectivas: la primera, desde el carácter global de los sistemas de los Estados-nación, donde estos son los actores políticos por excelencia en la escala global; la segunda, desde el rol del capitalismo como una influencia globalizadora fundamental que toca el orden económico; y la tercera, desde la creación de una comunidad científica global dentro de un constante flujo de información el cual permite la difusión de las ideas (1996). Si reflexionamos un poco sobre el apartado previo, podemos ver que las definiciones de Gibernau coinciden en su primera perspectiva de la globalización, con el señalamiento que hacía Wallerstein (citado en Serna, 2012). De igual manera, su segunda perspectiva coincide con lo que señalaban Ianni (1996) y Serna (2012) en relación con autores como Marx, Weber, Spencer y otros, quienes reconocían un proceso de circulación del capital en el mundo.

Otras definiciones de la globalización, como la de García (1999), nos dicen que esta es un "objeto no identificado", revelando la complejidad para analizar el fenómeno en sí mismo. García plantea que la globalización no es solo un conjunto de estrategias de grandes corporaciones por apropiarse de los recursos naturales y culturales de otros países, sino también, el horizonte imaginado por sujetos colectivos e individuales a fin de insertar sus productos en mercados más amplios (1999). En este sentido, podemos entender que el impacto cultural y social de la globalización, no radica solo en la transferencia cultural de los individuos y sus sociedades, sino en los nuevos significados que se generan a través de esta transferencia de rasgos, características y prácticas culturales.

Retomando a Brunner (citado en Ponce, 1998), a Gibernau (1996) y a García (1999), se podría coincidir entonces en el hecho de que los factores que hacen a la globalización del mundo actual particular, en relación con los procesos históricos en los que el mundo se ha interconectado, analizados desde la estructura formal del Estado-nación, son los factores tecnológicos que impactan directamente en las relaciones entre los individuos, pues estos permiten un flujo continuo y constante de información entre los actores sociales, quienes pueden llevar a cabo un intercambio de ideas que van cargadas de sus propias interpretaciones culturales de un mismo mundo. Este intercambio de ideas en espacios virtuales o territoriales da paso a la organización social transnacional que puede derivar en diferentes tipos de acción social, pero ya no necesariamente dentro de una estructura contenedora como la del Estado-nación. Esta aproximación permite entender también que los individuos, como integrantes de una sociedad, adquieren un rol activo en la caracterización de la globalización en el contexto actual, pues pese a no ser integrados directamente por el Estado o por los grupos de poder que toman las decisiones sobre las dinámicas nacionales de interacción global, síllevan a cabo acciones cotidianas que repercuten en la forma en la que un Estadonación enfrenta un proceso globalizatorio. 
Coincidiendo con que el proceso posmodernizatorio, como señala Bruner, es la expresión cultural del proceso de la globalización, podemos entonces decir que dependiendo de cada cultura el proceso globalizador tendrá una manifestación específica y peculiar, que algunos podrán coincidentemente llamar posmodernidad o no. Pero lo que resalta en esta reflexión es entender la importancia del aspecto social y cultural que no puede, por ningún motivo, minimizarse, obviarse, o no ser considerado importante ante el proceso globalizador.

Es en este punto donde cabe la reflexión acerca del proceso en el que la República de Corea ha enfrentado a la globalización, es decir, entender cómo en el contexto de la dinámica global, el país asiático ha podido integrarse a una dinámica no solo de intercambio comercial a partir de su crecimiento económico y político, sino también, cómo a nivel cultural y social se ha dado la inserción de esta sociedad a la dinámica global y cómo se ha interpretado desde su vida cotidiana el cambio o reajuste que demanda el proceso de globalización para su subsistencia como sociedad democrática, moderna, tecnologizada, apegada a las normas internacionales de seguridad social, etc.

\section{LA REPÚBLICA DE COREA} Y LA GLOBALIZACIÓN

Si partimos del hecho de que Corea es un país con una presencia internacional en la esfera política a través de organismos mundiales como la ONU, el G-20, la OCDE, por mencionar algunos, y sumamos la presencia económica que el país tiene cuando se ubica en la $15^{a}$ economía del mundo ${ }^{6}$, representada a través de sus grandes conglomerados que han logrado transnacionalizar sus productos a partir de diversos acuerdos comerciales con múltiples países, podemos entonces aseverar que el proceso globalizatorio enfrentado por el mundo ha sido un vehículo óptimo no solo para el desarrollo económico de Corea en los últimos años, sino que también ha servido para potencializar su posicionamiento como un actor político relevante en la escena mundial.

Según Samuel Kim (2000), es en 1995 que emerge una "fiebre por la globalización, de donde surge el término en coreano segyehwa", la palabra intentaba abrazar la dimensión política, cultural y social. Este proceso se da bajo el mandato del presidente Kim Young-sam, que al representar el primer Gobierno civil, generó reformas y nuevas estrategias para la administración del país. Como menciona Saxer,

...desde el 17 de noviembre de 1994, después de su participación en la cumbre de APEC, el presidente Kim Young-sam, anunció una política de globalización con el propósito explícito de hacer del país una nación avanzada.

La política tenía la propuesta de (1) crear una nación de primer nivel; (2) racionalizar todos los aspectos de la vida; (3) mantener la unidad nacional sobreponiéndola a las diferencias generacionales, regionales y de clase; (4) reforzar la identidad nacional

6 Según cifras del Banco Mundial en 2012 (The World Bank, 2017). 
de Corea como la base de una globalización exitosa; y (5) mejorar el sentido de comunidad con toda la humanidad. Para poder conseguir estas metas sería necesario mejorar la eficiencia económica promoviendo la autonomía, la competencia y la liberalización (s. f.).

Es en este periodo presidencial, según información de Kim, que se establece la necesidad de la democratización para poder participar en organismos internacionales como la OCDE, aseverando que las economías nacionales entrarían a la era de la economía mundial, una era de flujos de información global y de economías $\sin$ fronteras, donde la industria cultural llega a posicionarse como una de las más fundamentales (2000). También se le atribuye al Gobierno de Kim Dae-jung la aceleración del "proceso globalizatorio de Corea usando la demanda de Fondo Monetario Internacional para una reforma social y económica” (Shin, 2003).

La fuerza de la globalización coreana se inició en el Gobierno del presidente Kim Dae-jung, a través de un plan estratégico para la mejora del Estado de arriba hacia abajo, especialmente bajo la noción de democracia participativa y del mercado económico como mutuamente complementarios (Kim, 2000). Este modelo de Gobierno fue el que caracterizó el periodo del presidente Kim Dae-jung, quien trató arduamente de conciliar las relaciones entre ambas Coreas persiguiendo siempre la paz para la prosperidad de los pueblos. Esta búsqueda y promoción de la paz le confirió en el año 2000 el Premio Nobel de la Paz, el cual lo colocó en la escena internacional como un dirigente modelo y como un presidente conciliador con el Norte, que se planteó como fin la reunificación de la península. Este reco- nocimiento le proporcionó al presidente Kim la oportunidad de figurar de manera activa en el plano mundial, siendo reconocido por ser un presidente prodemocrático que apostaba al bienestar social.

Sin embargo, la importancia de la globalización para un país de comercio como Corea, lo forzó a una apertura con las exigencias de la competitividad global (Kim, 2000). No es de sorprender que el significado de segyehwa variara de grupo en grupo: fue un principio estratégico, un eslogan movilizador, una ideología hegemónica, o un nuevo emblema de la identidad nacional para un Estado que aspiraba a avanzar en el estatus de la clase mundial (Kim, 2000), factor que abrió la posibilidad de que cada sector interpretara el proceso de manera diferente. Es decir, pudo ser emitido desde el Estado con intenciones definidas, pero pudo también ser entendido y ejecutado de otra manera desde la sociedad misma.

En uno de los discursos presidenciales del año 2000, la visión del nuevo milenio, donde además de referirse a las transformaciones estructurales de la globalización, se enfatizaba la importancia de que los coreanos estuvieran preparados para adaptarse a los cambios (Kim, 2000), evidenciaba un discurso generalizador que en realidad no especificaba cuáles eran esos cambios para los que debían prepararse y, mucho menos, cómo cada individuo los enfrentaría. Por ejemplo, desde el sector religioso de la sociedad se percibe que:

La Iglesia católica jugó un rol importante en el viaje de Corea del Sur a la democracia, dando refugio a los disidentes y financiando a estudiantes activistas [...] El padre Park Dong-ho, dijo que el movimiento 
democrático de Corea del Sur no introdujo completamente valores como los derechos humanos y la justicia en la sociedad (Wall Street Journal, 2014).

Lo anterior evidencia que cada sector de la sociedad enfrentó el proceso tanto democrático como de la globalización de manera particular.

Así, es evidente que la entrada de Corea a la dinámica globalizadora desde el discurso oficial concentró sus esfuerzos en los aspectos que se consideraron convenientes para una estabilidad económica y un perfeccionamiento de sus prácticas políticas en el espacio internacional, es decir, el fenómeno de la globalización se percibió como una nueva dinámica mundial a la que tendrían que insertarse como país, como también menciona Shin (2003). Esto le sirvió a Corea para empoderarse desde la estructura estatal y lograr gestionar los espacios y los acuerdos con el exterior que generarían el llamativo desarrollo económico coreano y una base comercial fundamentada en el intercambio con otros países a través de las exportaciones. Hasta este punto, el Estado coreano percibía a la globalización, coincidiendo con Gibernau, desde el carácter global de los sistemas de los Estados-nación, donde estos son los actores políticos por excelencia en la escala global (1996). Estos se atribuían el poder de decidir sobre el rumbo de todo un país -que en la realidad es lo que le corresponde-, sin embargo, bajo el principio democrático, lo que queda en cuestión es la toma de decisión unilateral desde el Estado y la no inclusión de la sociedad, cuando las políticas estatales aspiraban a una democracia participativa, al menos desde el discurso oficial.
Es decir, que la República de Corea entendió la necesidad de gestionar desde el Estado hacia la arena internacional, de la misma manera que entendió la impostergable llegada del capitalismo globalizador y su potencial para afianzarse en los mercados exteriores a través de una red interconectada llamada mercado mundial. También, en esta modernización, Corea como Estado entendió la repercusión de los flujos de información transferidos a través de los desarrollos tecnológicos y del potencial que tendría la distribución de la información a través de las nuevas tecnologías.

Este proceso de inserción, desde el discurso modernizador, evidenciaría el éxito del país asiático en lo que refiere a su desarrollo económico y su capacidad política para gestionar nuevas alianzas y socios comerciales estratégicos, que es el discurso con el que se ha asociado a la República de Corea bajo el eslogan del "Milagro coreano" o "Milagro del río Han" en las últimas décadas. No obstante, desde la esfera social y cultural, este discurso no empata tan acordemente con la realidad y tampoco con la forma en la que los individuos coreanos enfrentan esta inserción en la cotidianidad.

Por ejemplo, Samuel Kim también menciona que, a pesar del creciente coro de globalización y globalismo, en el fondo Corea sigue sumida en el capullo del nacionalismo cultural exclusivo, el cual actúa como una poderosa y persistente restricción para este impulso de la globalización (2000), es decir, el proceso de la globalización impacta en las estructuras económica y política, pero la sociedad no lo vive en la misma dimensión. Kim (2000) señala que los coreanos siguen apropiando 
a la globalización como una meta nacional, es decir desde las estructuras del Estado, y reforzando sentimientos nacionalistas que se contraponen con el proceso de apertura social y cultural que la globalización presupone.

Por otro lado, Shin (2003) menciona, en oposición a la postura de Kim, que los coreanos no ven una contradicción inherente entre el nacionalismo y la globalización, pues él considera que en Corea existe una apropiación nacionalista de la globalización y que hay una intensificación de la identidad étnica como reacción al proceso de globalización (2003). Este argumento permite entender, por un lado, que la inserción de Corea al proceso globalizatorio sí fue planeada, inducida y ejecutada desde la estructura estatal y que, quizá como punto más importante, el resultado de este proceso impactó espontáneamente en la sociedad que, como respuesta a esta fuerza invisible, como señala Canclini, ha generado estrategias para entender el contexto en el que viven su realidad, las cuales se expresan en formas de resistencia como la intensificación de una identidad étnica basada en su imaginario de la pureza-racial de origen del pueblo coreano.

Desde el discurso oficial, donde se consideraron varios aspectos para el desarrollo de segyehwa, reconociendo, entre estos, "el valor estratégico de los coreanos en el exterior, especialmente los coreano-estadounidenses [...] promulgando una ley especial en el año
2000 para los coreanos viviendo en el exterior" (Shin, 2003). La intención de esta ley, como bien señala Shin, era reforzar la identidad étnica de los coreanos en el exterior y la globalización e interconexión básicamente a través del internet ${ }^{7}$. Lo que deja claro Shin (2003) en su estudio, como también lo señala Baker (2009), es la intención de reforzamiento de la identidad étnica que defiende Shin, una consecuencia de la estrategia del Gobierno coreano para su inserción al proceso de globalización.

Este tipo de medidas que se tomaron desde el Gobierno definitivamente fomentan que la sociedad coreana y el Estado coreano sigan confrontándose, es decir, que pese a sus políticas globalizadoras que reflejan un éxito económico y buenos alcances políticos, en el contexto social se siguen derivando una serie de actos ejecutados en la vida diaria de la sociedad coreana que permiten cuestionar directamente el éxito social de su política globalizadora.

Si partimos de que esa ley de reforzamiento de la identidad nacional, por citar alguno de los alcances sociales de estas políticas, se fundamenta en la identidad nacional de la posguerra de la península (1950-1953), donde se resalta el mito de origen del pueblo coreano conocido como mito de Tangun ${ }^{8}$, como el eje cohesionador de la sociedad coreana, podemos entender la reacción social a esta ley. Por ejemplo, en el mito de fundación se resaltan las cualidades biológicas-étnicas del pueblo coreano que le

$7 \quad$ Lo que llama la atención de esta ley es que solamente estaba dirigida hacia los coreano-estadounidenses, dejando excluidos al resto de los coreanos esparcidos por el mundo; menciona Shin que excluía específicamente a los coreanos étnicos de Rusia y China. Señala el autor que, posteriormente, la Suprema Corte de Corea resolvió como inconstitucional excluir a estos grupos étnicos coreanos del beneficio de la misma ley.

8 Para más información referente al Mito de Tangun, puede revisarse: Ilyon (1997). 
dan una particularidad humana-divina. La creencia de una descendencia del ancestro mitad dios-mitad humano sustenta la identidad étnica de la Corea actual, donde aún es común escuchar discursos de pureza racial entre los coreanos. Lo alarmante de este reforzamiento identitario es que lo coreano se superpone a la otredad, una otredad situada en individuos que se consideran como ciudadanos de segunda en esta sociedad asiática, como es el caso de los inmigrantes de países del sudeste asiático, o de países considerados pobres o en vías de desarrollo. Casos como el de los migrantes de países provenientes del sureste asiático, individuos de piel oscura o individuos de países que son considerados pobres o menos desarrollados que Corea, son algunos ejemplos por citar?

Se observa así que en los aspectos socioculturales en el proceso globalizatorio de Corea se seleccionaron elementos que en su momento se consideraron estratégicos. Sin embargo, es pertinente cuestionar cuáles elementos socioculturales son potencializados y cuáles no, para así entender desde qué perspectiva el planteamiento de la globalización para Corea consideraba a su sociedad portadora de su propia cultura, como un grupo con sujetos capaces de insertarse en una nueva dinámica que no solo traería cambios económicos y políticos estructurales al país, sino que requeriría de cambios fundamentales en la forma de percibir al mundo y de entablar relaciones con otras sociedades desde una plataforma democrática $\mathrm{y}$ de intercambio justo.

\section{CULTURA Y SOCIEDAD EN LA REPÚBLICA DE COREA, DESAFÍOS ANTE LA GLOBALIZACIÓN}

El proceso globalizatorio de la República de Corea, al igual que en otras sociedades, está lleno de anhelos y contradicciones. Uno de los grandes retos de la globalización es, sin duda, generar espacios de interacción bajo procesos democráticos en un entorno de respeto en la realidad social.

Hemos mencionado que, en la actualidad, las sociedades modernas enfrentan grandes dificultades de la globalización dinamizada por la liberación de los mercados, que son institucionalizados en la estructura política y ejecutados desde la estructura económica. Estas acciones pueden satisfacer las expectativas de los grupos que incentivan los acuerdos políticos y comerciales en el mundo, pero quizá, uno de los grandes fallos del mundo globalizado, es su impacto en las sociedades y sus culturas, pues, las interacciones que se dan entre los grupos humanos generan realidades desiguales $\mathrm{y}$, muchas veces, de desventaja para algunos grupos sociales o individuos.

\footnotetext{
9 La migración a Corea se ha generado por la necesidad de mano de obra barata, para suplir las necesidades de diversos sectores como el industrial y el agrícola. Las vacantes de trabajo son espacios laborales que generalmente los coreanos y coreanas no desean hacer porque están sobrecalificados para ellos o el pago no es representativo para el ingreso coreano. Estos trabajos que requieren más trabajadores, integran a inmigrantes de países considerados pobres o menos desarrollados, como Camboya, Tailandia, Filipinas, Vietnam, Pakistán, India, Indonesia, entre otros. Para más referencias véase Jack Board (2015).
} 
Como menciona García:

La globalización [...] tiene una complejidad multidimensional: en la globalización convergen procesos económicos, financieros, comunicacionales y migratorios que acentúan la interdependencia entre distintas clases sociales, y generan mayor interconexión supranacional que en cualquier época anterior. Es un proceso abierto, que incluye diversas tendencias y efectos, a veces combinables, a veces contradictorios, dentro de una misma sociedad (2002).

Therborn prefiere hablar de globalizaciones, reconociendo los múltiples procesos sociales implícitos en este fenómeno mundial (citado en Serna, 2012), enfatizando la pluralidad de perspectivas que se deben considerar para integrar no solamente a las estructuras gubernamentales y económicas, sino a las estructuras sociales y su cultura a estos procesos globalizatorios.

El caso de la República de Corea es un buen ejemplo de la realidad que García señala en su discusión teórica cuando hace referencia a la multiplicidad de efectos que se generan a través de la globalización. En Corea del Sur, por ejemplo, se analiza desde la parte estruc- tural, por referirnos a la política y la economía, cómo la globalización ha beneficiado el desarrollo del país. Por ejemplo, Shin (2003) mencionaba que Corea es un país que no se ha conflictuado con el proceso globalizatorio, por el contrario, ha sabido aprovechar las bondades del fenómeno mundial y que esto, contrariamente a lo que se argumenta en otros casos, ha permitido reforzar la identidad étnica a través de una apropiación nacionalista de la globalización, como ya se había señalado en el apartado anterior. Sin embargo enfatiza claramente, reforzando el argumento que cita de Appadurai (1990), que "Mientras la globalización produce presiones para la 'convergencia', sus efectos son indirectos, mediados por los políticos y las políticas domésticas. En particular, el Estado aun juega un rol proactivo en el modelado del proceso de globalización" (Shin, 2003). Apuntando claramente al vacío de representatividad que tiene el conjunto social de Corea. Con esto no descalificamos la organización de la sociedad civil coreana, pues incluso recientemente esta demandó, de una manera activa y participativa, la renuncia de la presidenta Park Geun Hye ${ }^{10}$, la cual ya ha sido destituida por cargos de corrupción.

10 El periodo presidencial de Park Geun Hye estuvo marcado por una clara división de la sociedad, la parte que estaba a favor de su mandato y quienes se oponían. Sin embargo, recientemente la sociedad exigió su renuncia. Los motivos son diversos, pero quizá el detonante de esta protesta social fue el escándalo donde "la presidenta le confería favores financieros a su amiga y chamana Choi Soon-sil” (Tai, 2016). También en este reportaje, el portal de noticias sCMP señala que "Tres grandes protestas han tenido lugar hasta ahora, cada una mayor que la anterior. En el evento de la semana pasada, los participantes incluyeron sindicatos de trabajadores, sindicatos de estudiantes universitarios, sindicatos de agricultores, partidos de izquierda, familias del desastre del ferry Sewol, grupos de feministas y ciudadanos de todos los sectores de la vida [...] Corea del Sur también enfrenta [...] problemas específicos de cultura. Una combinación de normas sociales arcáicas e instituciones corruptas, han contribuido a los graves problemas de la nación de desigualdades económicas, sociales y de género" (Tai, 2016). 
Shin (2003) también reconoce que específicamente países del este de Asia, como China, Corea y Japón, históricamente han adoptado los beneficios de Occidente para el reforzamiento de sus propios Estados, aseveración que naturaliza la hegemonía del pensamiento occidental sobre otras regiones del mundo, en este caso, del noreste de Asia, y el cual enfatiza también las bondades de la globalización cuando es integrada por países que saben ver oportunidades de desarrollo en la dinámica globalizante; lo que se le escapa explicar son las políticas unilaterales a las que dichos países han recurrido para implementar estos cambios en sus realidades, dejando de lado el aspecto democratizador y de participación integral por cada integrante de sus sociedades, garantizando la igualdad de oportunidades y la equidad social.

Los grupos de poder que pusieron en marcha el proceso globalizatorio de Corea, tanto Gobierno como empresarios ${ }^{11}$, impusieron una narrativa a través del discurso del ideal de la modernidad que invitaba a la sociedad a cambiar formas y prácticas cotidianas que, probablemente, no fueron evidentes en los primeros años, pero que hoy han desembocado en escenarios socioculturales ambiguos, no solo al interior de la sociedad coreana, sino también en sus relaciones con el exterior. Estas realidades no solo impactan en la vida cotidiana, como en muchas otras sociedades tocadas por el neoliberalismo discursado de globalización, sino que han permitido que, en la búsqueda de nuevos mercados y socios comerciales para Corea, el Estado y la élite económica modelen una imagen del país que no corresponde a las características identitarias que definen y representan a la sociedad coreana y, en consecuencia, al país, como es el caso de la imagen que se transmite a través de la cultura pop generada desde la industria del entretenimiento ${ }^{12}$.

Además de provocar, al interior de la sociedad, reacciones que polarizan los posicionamientos de sus individuos, dando paso a discursos y practicas discriminatorias, marginalizantes o no inclusivas hacia personas de diferente nacionalidad o condición sociocultural, pues la sociedad local no ha sido capaz de procesar los cambios sociales que demandaba el proceso globalizatorio en la vida cotidiana y no solo en el discurso.

La ambigüedad con la que se manifiesta la realidad impactada por la globalización en la sociedad coreana refleja, por un lado, el discurso de un país modelo y ejemplar en el que el proceso de desarrollo económico ha aportado al crecimiento y la estabilidad política y

11 Los chaebols, o conglomerados coreanos, son grupos empresariales con múltiples diversificaciones que conforman un amplio sector del mercado surcoreano. Desde el inicio de la República de Corea, el Gobierno ha favorecido estos grupos que se organizan primordialmente a través de redes familiares o lazos de parentesco. Estos grupos, que concentran un gran porcentaje de la actividad económica coreana, han sido frecuentemente subsidiados por el gobierno en épocas de crisis, cuestión que ha evidenciado la inequidad en la distribución de los recursos del Estado para todos los sectores del país. Para más información véase Lee (2000, pp. 1-20).

12 Para más información referente a la cultura pop de Corea, a la Ola Coreana o al Hallyu, véase: Lopez y Ryzhkov (2017, pp. 131-158), y López (2015, pp. 171-195). 
social, pero por otro lado, subraya las carencias socioculturales para entender contextos ajenos y diferentes a través de la adaptación y el diálogo intercultural.

Una de las grandes contradicciones de este proceso globalizador coreano es no haber atendido en su justa dimensión las implicaciones del cambio sociocultural en la sociedad coreana. Era lógico avizorar una influencia cultural de Occidente, como han señalado Berger y Huntington (2002) en relación con la cultura occidental dominante en el proceso de globalización, la que propiciaría una mezcla de elementos provenientes de otras culturas, aquellas que dominan la forma de percibir al mundo y sus realidades en el entorno globalizado, pues estos son de hecho los cambios a los que se tendría que enfrentar la sociedad coreana, más allá de ser a los que se refería el Gobierno cuando desde su discurso exhortaba a sus ciudadanos a estar listos.

Lo que queda claro es que hay una gran contradicción entre la invitación del Gobierno para preparar a sus ciudadanos ante estos cambios, pero por otro lado, no entender que habría un choque cultural que tendría como resultado una hibridación de la cultura local. $\mathrm{Al}$ mismo tiempo, si se considera este proceso de adaptación o aculturación que arroja como resultado la hibridación cultural, entonces ¿cómo entender el reforzamiento de una identidad basada en la pureza? Y sobre todo, ¿para qué fomentarla si lo que en realidad se buscaba era la adaptación de la sociedad a los cambios por venir?

La hibridación o adopción de elementos culturales del exterior reinterpretados de manera local en Corea ha sido, como señala Gibernau (2007), aleatoria. Pero sin duda esta hibridación, según García (2009), le ha otorgado a Corea las características para interactuar en el espacio global. Lo que permite nuevamente cuestionar ¿por qué existen prácticas sociales que marginan, discriminan o que acentúan la pobreza en unos y la riqueza en otros, en una sociedad que sigue siendo promovida a través de un discurso de éxito y ejemplo en el escenario global?

No se puede criticar el proceso particular por el que ha pasado Corea en su inserción al escenario global, pues cada país, dentro de sus propios contextos, genera características particulares válidas desde su realidad, reconociendo, como menciona Therborn, los múltiples procesos sociales implícitos en este fenómeno. Sin embargo, existen situaciones pendientes que obligan al análisis y a la reflexión, sobre todo cuando se postula una experiencia particular como un modelo de éxito que debe ser imitado por otras sociedades. Temas relevantes como la inequidad de ingresos ${ }^{13}$, la representación femenina en la política ${ }^{14}$, la violencia contra las mujeres ${ }^{15}$, el rechazo a los inmigrantes o extranjeros, la inequidad de oportunidades

13 Inequidad de ingresos 0.302 donde 0 es equidad completa y 1 inequidad completa (OECD, 2017a).

14 Mujeres con voz en la política: $16 \%$ en 2014, cifras de la ocDE (2017c).

15 Violencia contra la mujer: 19\% en 2014, cifras de la ocDE (2017b). 
para los jóvenes, la corrupción, el incremento de la pobreza, etc., son temas sensibles que merecen ser atendidos desde la reflexión y el análisis social y humano.

\section{CONCLUSIONES}

Diversas consideraciones para tomar en cuenta aparecen cuando se hace una aproximación al estudio de los cambios socioculturales generados en una sociedad a partir de su inserción al proceso globalizador.

Como se ha mencionado a lo largo de este trabajo, la República de Corea se inserta en el proceso de la globalización a partir de cambios estructurales que, desde las élites del poder político y económico, fluyeron rápidamente hacia la masa social, para iniciar su inserción en la globalización.

Este proceso coincide con dos de las tres perspectivas que señala Gibernau. La primera, desde el carácter global de los sistemas de los Estados-nación, donde estos son los actores políticos por excelencia en la escala global; la segunda, desde el rol del capitalismo como una influencia globalizadora fundamental que toca el orden económico.

Pero el caso particular de Corea, que probablemente se replique en otras sociedades, invita a analizar la globalización como la define García, entendiéndola no solo como un conjunto de estrategias de grandes corporaciones por apropiarse de los recursos naturales y culturales de otros países, sino también, el horizonte imaginado por sujetos colectivos e individuales (1999). Desde esta perspectiva rescatamos la necesidad primaria de entender estos procesos en los que el mundo se interconectó en todo su contexto, es decir, dejar de atender a ellos a través de una sola visión promovida por intereses unilaterales o de élites, y visualizar que en tanto sean procesos que impliquen a los grupos sociales, estos deben ser considerados, tomados en cuenta, escuchados, con derecho a opinar sobre esos procesos para que el multidimensionalismo que implica el proceso de la globalización pueda acoplarse a la estructura de cada sociedad y su cultura y no, por el contrario, que cada sociedad se adapte al proceso globalizante.

En el proceso particular de la República de Corea en torno a la globalización se evidencian principios locales que trataron de adaptar la dinámica que el mundo exterior imponía de a poco a diversas sociedades. Según el término coreano para definir la globalización -segyehwa-descrito por Samuel Kim (2000), implica el intento que desde el Estado coreano hubo para retomar los puntos cardinales del proceso globalizatorio al que tuvieron que insertarse en la dinámica mundial, razón por la cual el propio término intentó describir no solo las implicaciones políticas, sino también las culturales y sociales a las que la República tendría que enfrentarse. Las cinco metas establecidas para el proceso globalizatorio que se implementaría en Corea, que se han mencionado, consideraron:

1) crear una nación de primer nivel; 2) racionalizar todos los aspectos de la vida; 3) mantener la unidad nacional sobreponiéndola a las diferencias generacionales, regionales y de clase; 4) reforzar la identidad nacional de Corea como la base de una globalización 
exitosa; y 5) mejorar el sentido de comunidad con toda la humanidad (Saxer, s. f.).

Pero estas metas estuvieron subsumidas a la mejora de la eficiencia económica la cual promovería la autonomía, la competencia y la liberalización.

Sin embargo, aunque en el discurso se perciben claramente elementos sociales y culturales en todos los puntos, las metas que se establecieron desde un discurso nacionalista distarían sobremanera de la realidad a la que la República de Corea se enfrentaría al poner en marcha su meta principal: la liberalización de su economía. Así, en la meta número tres se resalta la unidad nacional por encima de las diferencias generacionales, factor que evidentemente no se ha logrado. Un ejemplo de ello fue la elección de la última presidenta, quien logró su victoria justamente gracias a las diferencias generacionales que existen aún en la sociedad coreana, donde claramente los adultos mayores y los jóvenes se confrontan por perspectivas opuestas sobre la realidad social de su país. Igualmente, la meta cuatro, reforzar la identidad nacional para una globalización exitosa, punto ya tratado en apartados anteriores, no ha logrado tampoco su cometido pues al existir desigualdad entre los mismos grupos de coreanos por su lugar de nacimiento o su mezcla con otros grupos, se puede hablar de un efecto contraproducente en el reforzamiento de una identidad nacional la cual descarta la otredad. A partir de la meta anterior, la meta cinco evidentemente no logra su cometido, pues aunque el Estado coreano ha implementado estrategias que hablan de multiculturalismo y aceptación de actores no coreanos en su socie- dad, en la realidad actual aún hay elementos que evidencian la discriminación y la fobia por personas de origen distinto al coreano.

Quizá una razón para las metas fallidas es justamente el último punto que resalta Saxer (s.f.), pues al dar prevalencia a la eficiencia económica que promovió la autonomía, la competencia y la liberalización, se priorizó nuevamente el factor estructural dejando en un plano secundario elementos fundamentales para un proceso globalizatorio en el que se consideraran a conciencia las implicaciones socioculturales.

Como menciona Samuel Kim, no es de sorprender que el significado de segyehwa variara de grupo en grupo: fue un principio estratégico, un eslogan movilizador, una ideología hegemónica, o un nuevo emblema de la identidad nacional para un Estado que aspiraba a avanzar en el estatus de la clase mundial; la importancia de la globalización para un país de comercio como Corea lo forzó a una apertura con las exigencias de la competitividad global (2000). Lo cual nos permite entender que aunque Corea, desde el discurso oficial, interpretó el proceso de globalización al cual tendrían que insertarse en conjunto con su sociedad, en la realidad aspiraba a lograr, al menos, la internacionalización económica que pudiera dar seguridad y autonomía a un país que no estaba socialmente preparado para una transformación más allá de lo económico, pero que sí necesitaba prepararse para enfrentar la dinámica de la liberalización de los mercados promovida por la globalización.

Así, podemos entender que el proceso de globalización por el cual Corea ha transitado es similar al de otros países. Corea intentó definir 
en su propio idioma lo que la globalización implicaba, sin embrago, el ajuste social y cultural que este proceso requiere no ha logrado reflejarse en varios aspectos que se consideran fundamentales para lograr una sociedad equitativa, justa, respetuosa e incluyente, todos estos, aspectos que se persiguen de la mano de un país desarrollado.

El proceso globalizatorio nos arroja como individuos a reinterpretar la realidad a partir de las restructuraciones físicas, políticas, económicas, culturales e ideológicas que se han propiciado por lo que denomina García un capitalismo transnacionalizado. Los retos a los que nos enfrentamos como individuos y sociedades son el entendimiento de un cambio social que se da de manera más vertiginosa y el desarrollo pronto de las estrategias de adaptación que nos permitan sobrevivir en la realidad globalizada.

En el caso particular de la República de Corea, se ha mencionado que las prioridades establecidas por el Estado para enfrentar la globalización quedaron lejos de la sociedad, es decir, las estrategias que se implementaron para enfrentar el proceso de la globalización se dieron desde las élites políticas y empresariales, dejando que la sociedad enfrentara estos procesos de la manera que su propia interpretación del fenómeno le permitiera. Esto desembocó en diversos conflictos a nivel social y cultural, como se ha señalado.

Cómo enfrentar los procesos migratorios, el empoderamiento de la mujer, la necesidad de integrarla en la esfera productiva como mano de obra profesional y capacitada, el envejecimiento poblacional, etc.

En este proceso, la República de Corea ha priorizado el desarrollo de los sectores económico-empresarial, tecnológico y político. Estos, sin duda, han puesto al país en la arena internacional a la vista del mundo pero, por otro lado, la esfera sociocultural no ha sido integrada de una manera completa. Es decir, se tomaron de esta los elementos necesarios de manera selectiva para respaldar el proyecto planteado desde el Estado, pero se generaron también choques entre sociedad y Estado que evidencian que el país asiático no ha podido adaptarse al proceso global de manera óptima.

Temas como la xenofobia en contraposición al nacionalismo étnico, la inequidad de ingresos en contraposición a la riqueza de los conglomerados, la violencia contra la mujer en contraposición a la perpetuidad de la sobrevaloración del hombre respaldada por el confucianismo, la inequidad del acceso a la educación en contraposición con la educación en instituciones privilegiadas, la amplitud de la brecha entre el poder adquisitivo de la clase media y la alta, la representatividad de mujeres en el poder en contraposición a la mayoría de los hombres, etc., son temas importantes de atender en el contexto actual de la sociedad coreana. No se puede apelar a ser un país modelo, ejemplo del desarrollo exitoso, cuando existen conflictos y desacuerdos sociales en un país democrático. El ideal sigue siendo, sin duda, apelar a una globalización más humana y justa, que rescate los aciertos y atienda los errores.

\section{REFERENCIAS}

Appadurai, A. (1999). Disjuncture and Difference in the Global Cultural Economy, en Global Culture (pp. 295-310). London: Sage Publications. 
Baker, D. L. (2009). Societal Cohesion, en Korea Confronts Globalization, Routledge Advances in Korean Studies (pp. 227-228). London: Routledge.

Berger, P. L. y Huntington, S. (2002). Many Globalizations, Cultural Diversity in Contemporary World. New York: Oxford University Press.

Board, J. (2015). Migrant workers treated like "slaves" in South Korea's agricultural industry. Channel New Asia. Recuperado de http://www.channelnewsasia.com/news/asiapacific/migrant-workerstreated/1872922.html

Channel New Asia (2015). Migrant workers treated like "slaves" in South Korea's agricultural industry. Recuperado de http://www.channelnewsasia. com/news/asiapacific/migrant-workers-treated/1872922.html

García, N. (1999). La globalización imaginada. Buenos Aires: Paidós.

García,, N. (2002). Culturas populares en el capitalismo. México: Grijalbo.

García, N. (2009). Culturas híbridas. Estrategias para entrar y salir de la modernidad. México: Random House Mondadori.

Guibernau, M. (1996). Nationalisms: The Nation-State and Nationalism in the Twentieth Century. Oxford: Polity Press.

Hahm, C. (2008). South Korea’s Miraculous Democracy. The Journal of Democracy, 19 (3), 128-142.

Ianni, O. (1996). Teorías de la globalización. México: Siglo xxi Editores, CEIICH-UNAM.

Ilyon (1997). Samguk Yusa: Legends and History of the Three Kingdoms of Ancient Korea. Seoul: Yonsei University Press.

Kim, D. (2015). How Jasmine Lee, One od Most Hated Woman in Korea, Is Changing the Country. Huff Post Korea. Recuperado de http://www. huffingtonpost.com/2015/05/07/jasmine-leekorea_n_7214186.html
Kim, S. (2000). Korea and Globalization. New York: Columbia University. Recuperado de http:// www.koreasociety.org/doc_view/357-korea-andglobalization-by-samuel-kim

Kim, Y. S. (1996). Korea's Reform and Globalization. Seoul: Korean Overseas Information Service.

Lee, P.-S. (2000). Economic Crisis. Discussion Paper Series, 14, 1-20.

Lee, Y.-i. (2012). Soulth Korea's globalization in the late twentieth century: An end to economic nationalism?, en Globalization and Economic $\mathrm{Na}$ tionalism in Asia (pp. 157-176). Oxford: Oxford University Press.

López R., N. (2015). El rol del Hallyu como cultura pop en la creación y la difusión de la imagen de la mujer coreana contemporánea. PORTES, revista mexicana de estudios sobre la Cuenca del Pacifico, 9 (18), 171-195.

López R., N. y Ryzhkov, A. (2017). Hallyu as a vehicle for internationalizing the Korean culture: official discourse and its repercussions. Journal of Korean Culture, 36, 131-158.

Nelson, R. y Pack, H. (1999). The asian miracle and modern growth theory. The Economic Journal, 109 (Issue 457), 416-436.

OECD (2017a). Income Inequality (Indicator). doi: 10.1787/459aa7f1-en Recuperado de https:// data.oecd.org/inequality/income-inequality. htm\#indicator-chart

OECD (2017b). Violence Against Women (Indicator). doi: 10.1787/f1eb4876-en Recuperado de https:// data.oecd.org/inequality/violence-against-women.htm

OECD (2017c). Women Political Voice (Indicator). doi: 10.1787/edc3ff4f-en Recuperado de https:// data.oecd.org/inequality/women-political-voice. htm\#indicator-chart 
Oh, I. y Jun, H. (2016). Economic Miracle: from pos-war reconstruction to post-crisis affluence. Routledge Handbook of Modern Korean History (pp. 295313). New York: Routledge.

Ponce de la F., H. (1998-1999). José Joaquín Bunner: globalización cultural y posmodernidad. Revista Chilena de Humanidades, 18/19, 313-318.

Saxer, C. J. (s/f.). Globalization as Policy: The South Korean experience. Documento no publicado.

Serna de la G., J. M. (2012). Impacto e implicaciones constitucionales de la globalización en el sistema jurídico mexicano. México: unam.

Shin, G.-W. (2003). The Paradox of Korean Globalization. California: Stanford University-The Asia/Pacific Research Center.
Strother, J. (2014). South Korea's protesting priests. The Wall Street Journal. Recuperado de http:// blogs.wsj.com/korearealtime/2014/08/15/southkoreas-protesting-priests/

Tai, C. (2016). South Korea's umbrella movement? How protests against park Geun-hye exposed crack in a 'slave' democracy. SCMP. Recuperado de http://www.scmp.com/week-asia/politics/ article/2046970/how-mass-protests-south-koreaexposed-cracks-its-slave-democracy

The World Bank (2017). Overview (Republic of Korea). The World Bank. Recuperado de http://www.worldbank.org/en/country/korea/overview. 